\title{
Diclofenac Induced Fixed Drug Eruption
}

\author{
M Natesh Prabhu' ${ }^{1 *}$, Akshay Khumar² and Deva Kumar ${ }^{1}$ \\ ${ }^{1}$ Department of Pharmacology, Dhanalakshmi Srinivasan Medical College and Hospital, India \\ ${ }^{2}$ Dhanalakshmi Srinivasan Medical College and Hospital, India
}

Submission: November 21, 2016; Published: November 30, 2016

*Corresponding author: M Natesh Prabhu, Department of Pharmacology, Dhanalakshmi Srinivasan Medical College and Hospital, Perambalur-621212, Tamilnadu, India, Email: drnateshprabhu@gmail.com

\begin{abstract}
Fixed drug eruptions (FDE) are common dermatological adverse drug reactions which are commonly caused by antimicrobials, anticonvulsants and non-steroidal anti-inflammatory drugs. It is a clinical condition occurring in the same site/sites each time when the drug is administered. Here we present a case of diclofenac induced fixed drug eruption with a Naranjo scoring of 6 (Probable). The patient recovered after stopping diclofenac. This projects the importance of ADR monitoring which is a part of Pharmacovigilance Programme of India (PvPI).
\end{abstract}

Keywords: FDE; Fixed Drug Eruption; Diclofenac; PvPI; ADR; Pharmacovigilance; Naranjo scale

Abbreviations: FDE: Fixed Drug Eruptions; PvPI: Pharmacovigilance Programme of India; ADR: Adverse Drug Reactions

\section{Introduction}

Adverse Drug Reactions (ADR) are defined as a noxious response to a drug which is unintended and which occurs at doses normally used in man for prophylaxis, diagnosis, or therapy of disease or for modification of physiological function [1]. The main pillar of post marketing surveillance is the spontaneous reporting of ADR [2]. In India Pharmacovigilance Program of India (PvPI) improve patient safety and welfare in Indian population by monitoring drug safety and thereby reducing the risk associated with use of medicines [3]. Fixed drug eruptions (FDE) are common dermatological adverse drug reactions which are commonly caused by antimicrobials, anticonvulsants and non-steroidal anti-inflammatory drugs. It is a clinical condition occurring in the same site/sites each time when the drug is administered [4].

\section{Case scenario}

A 42 year old male patient complained of erythema over both palms, blisters over the genitals and swelling of both the lower limbs and fever (1010 F). The patient is a known case of Type 2 Diabetes Mellitus since 2 years. On examination, multiple bullae with raw erythema and desquamation were seen over the abdomen, both the lower limbs and groin. Multiple ulcers were present over the groin due to scratching by the patient (Figure 1). The patient gave a history of consuming oral diclofenac tablets, over the counter, for tooth ache a couple of days before he developed these skin lesions. No previous history of similar reaction. Investigations were done and the results are as follows (Table 1). An opinion from the Department of Medicine was sought. Inj. Insulin HR 8 units BD and Inj. Insulin Isophane 6 Units BD were given to control his diabetes. Further Tab. Roxithromycin, Dexamethasone ointment, Tab. Chlorpheneramine maleate, Tab. Cetrizine, Tab. Rantac and Tab. Bifillac were prescribed. The fasting and post prandial blood glucose were brought under control. The patient's condition improved and he was discharged after 14 days and advised a review after 2 weeks.

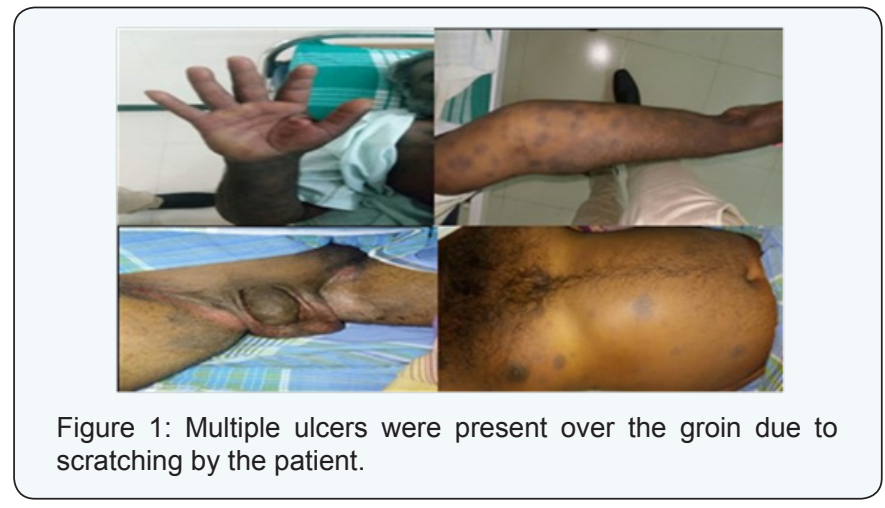


Table 1: The patient gave a history of consuming oral diclofenac tablets, over the counter, for tooth ache a couple of days before he developed these skin lesions. No previous history of similar reaction. Investigation was done and the results are as follows.

\begin{tabular}{|c|c|c|}
\hline S. No & Investigation & Result \\
\hline 1 & Haemoglobin & $1.2 \mathrm{~g} / \mathrm{dl}$ \\
\hline 2 & Sr. Creatinine $/ \mathrm{dl}$ \\
\hline 3 & Sr. Uric acid & $5.3 \mathrm{mg} / \mathrm{dl}$ \\
\hline 4 & RBS & $160 \mathrm{mg} / \mathrm{dl}$ \\
\hline 5 & PPBS & $301 \mathrm{mg} / \mathrm{dl}$ \\
\hline 6 & LFT & WNL \\
\hline 7 & RFT & WNL \\
\hline
\end{tabular}

RBS: Random Blood Sugar; PPBS: Post Prandial Blood Sugar; LFT: Liver Function Test; RFT: Renal Function Test; WNL: Within Normal Limits.

\section{Discussion}

The patient was found to be allergic to diclofenac. Rechallenge test was not done and the condition did not aggravate when the patient was given Paracetamol to treat the fever. The casualty assessment was done using Naranjo Scale [5] and the score was 6: Probable ADR - The reaction

i. followed a reasonable temporal sequence after a drug,

ii. followed a recognized response to the suspected drug,

iii. Was confirmed by withdrawal but not by exposure to the drug, and

iv. Could not be reasonably explained by the known characteristics of the patient's clinical state.

\section{Conclusion}

Diclofenac can be the probable cause of FDE in this patient.

\section{References}

1. http://www.ipc.gov.in/PvPI/pv_faq.html.

2. Kalaiselvan V, Prasad T, Bisht A, Singh S, Singh GN (2014) Adverse drug reactions reporting culture in Pharmacovigilance Programme of India. Indian J Med Res 140(4): 563-564.

3. http: //www.ipc.gov.in/PvPI/pv_about.html.

4. RR Bhosale (2013) Diclofenac Induced Fixed Drug Eruptions-A case report. International Journal of Basic and Applied Chemical Sciences 3(1): 60-61.

5. Naranjo CA, Busto U, Sellers EM, Sandor P, Ruiz I, et al. (1981) A method for estimating the probability of adverse drug reactions. Clin Pharmacol Ther 30(2): 239-245.

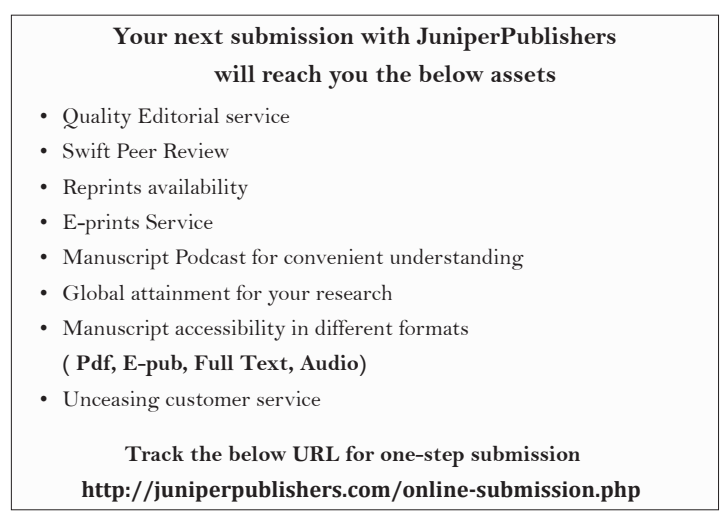

